\title{
Salix transect of Europe: patterns in the most abundant chrysomelid beetle (Coleoptera: Chrysomelidae) herbivores of willow from Greece to Arctic Norway
}

Roy Canty ${ }^{\ddagger}$, Enrico Ruzzier ${ }^{\ddagger}$, Quentin Cronk ${ }^{\S}$, Diana Percy ${ }^{\ddagger}$

‡ Natural History Museum, London, United Kingdom

$\S$ University of British Columbia, Vancouver, Canada

Corresponding author: Quentin Cronk (quentin.cronk@ubc.ca), Diana Percy (d.percy@nhm.ac.uk)

Academic editor: Lyubomir Penev

Received: 16 Aug 2016 | Accepted: 20 Sep 2016 | Published: 28 Sep 2016

Citation: Canty R, Ruzzier E, Cronk Q, Percy D (2016) Salix transect of Europe: patterns in the most abundant chrysomelid beetle (Coleoptera: Chrysomelidae) herbivores of willow from Greece to Arctic Norway. Biodiversity Data Journal 4: e10194. doi: 10.3897/BDJ.4.e10194

\section{Abstract \\ Background}

Chrysomelid beetles associated with willow (Salix spp.) were surveyed at 41 sites across Europe, from Greece (lat. $38.8^{\circ} \mathrm{N}$ ) to arctic Norway (lat. $69.7^{\circ} \mathrm{N}$ ).

\section{New information}

In all, 34 willow-associated chrysomelid species were encountered, of which eight were very abundant. The abundant species were: Crepidodera aurata Marsham, 1802 at 27 sites, Phratora vitellinae (Linnaeus, 1758) at 21 sites, Galerucella lineola (Fabricius, 1781) at 19 sites, Crepidodera fulvicornis (Fabricius, 1792) at 19 sites, Plagiodera versicolora (Laicharting, 1781) at 11 sites, Crepidodera plutus (Latreille, 1804) at nine sites, Chrysomela vigintipunctata Scopoli, 1763 at nine sites and Gonioctena pallida (Linnaeus, 
1758) at eight sites. The mean number of willow associated chrysomelid morphospecies at each site was 4.2 . Around $20 \%$ of the total variance in chrysomelid distribution could be accounted for by latitude, but this is mainly due to distinctive occurrence patterns at the northern and southern parts of the transect. There was a paucity of chrysomelids at Greek sites and a distinctively northern faunal composition at sites north of Poland. Considerable site-to-site variation in colour was noted, except in $G$. lineola, which was chromatically invariant.

\section{Keywords}

Salicophagy, salicivorous insects, Salicaceae, Chrysomelidae, Europe, megatransect

\section{Introduction}

Chrysomelidae Latreille, 1802, commonly known as leaf beetles, make up a very large and important major group of phytophagous beetles (Jolivet and Verma 2002). This family is divided in twelve subfamilies (Haddad and McKenna 2016) and more than twenty tribes but our study mainly focuses on the following three tribes, abundant in our collections: Chrysomelini Latreille, 1802 (Sub. Chrysomelinae Latreille 1802), Galerucini Latreille, 1802 and Alticini Newman, 1834 (Sub. Galerucinae Latreille, 1802). They range from host plant specialists (Jurado-Rivera et al. 2009) to generalist herbivores and many species are recorded feeding on willows.

Willows (Salix spp.) are trees and shrubs widespread in N. temperate regions, extending into boreal and arctic habitats. As they are abundant and widespread they form an important food source for specialist and generalist herbivores of all kinds, and are thus ecological "foundation" species (Cronk et al. 2015). Willow feeders include a number of generalist and specialist chrysomelids, which are of great interest for a number of reasons, as set out below.

First, willow-feeding chrysomelids are economically important pests. Willows are a traditional crop for basket making, and more recently they have been extensively planted in both North America and northern Europe as biomass energy crops. Chrysomelids are potentially destructive pests of such plantations (Larsson and Wirén 1982; Royle and Ostry 1995) with Phratora vulgatissima (blue willow beetle), P. vitellinae (brassy willow beetle) and Galerucella lineola (brown willow beetle) being the major pest species reported.

Secondly, there is considerable variation in the susceptibility of different willows to beetle attack (Hodkinson et al. 1998; Kendall et al. 1996), mirrored by differing feeding preferences of beetles for different willows (Rowell-Rahier 1984; Kelly and Curry 1991b). For instance in Britain, P. vulgatissima, while present on most willows, avoids Salix gmelinii (syn. S. burjatica, S. dasyclados Wimm.) and Salix $\times$ mollissima (S. triandra $\times$ S. viminalis) (Sage and Tucker 1998). In a study which tested preference by $P$. vulgatissima for the 
segregating progeny of the cross $S$. gmelinii $\times$ S. viminalis, a great variation in herbivore performance (survival and oviposition success) was found (Torp et al. 2013). Kelly and Curry $1991 \mathrm{~b}$ have shown that the resistance of $S$. gmelinii to herbivory by $P$. vulgatissima is likely due to the high amounts of the toxic phenolglycoside (salicylate) salicortin in the plant. Phenolglycoside occurrence varies greatly in willows, occurring in S. nigricans, $S$. purpurea and $S$. fragilis but absent in $S$. alba, $S$. caprea and $S$. cinerea (Rowell-Rahier 1984) and it has been suggested that the presence of toxic phenolglycosides promotes host specificity among herbivores (Rowell-Rahier 1984), while deterring generalist such as P. vulgatissima and $G$. lineola (Kendall et al. 1996). All this suggests that there is a complex co-evolutionary context existing between willows and their herbivorous beetles, mediated by plant biochemistry.

Thirdly, willow-feeding chrysomelids have a remarkable chemical ecology in which the larvae of the beetles use plant-derived chemicals for defence (Boland 2015; Pasteels et al. 1988). It is postulated that defence in these beetles was originally through purely endogenously-synthesized chemicals, but adaptation to feeding on highly toxic willow hosts facilitated a transfer to plant-derived molecules (Pasteels et al. 1990). When attacked, chrysomelid larvae discharge toxic droplets from glandular reservoirs on their backs. These glands have been called "bioreactors" (Boland 2015) as they perform final steps of toxin synthesis from plant chemicals trafficked into the glands by an intricate molecular transport system. For instance $P$. vitellinae secretes a copious amount of salicylaldehyde (Pasteels et al. 1982; Pasteels and Gregoire 1984). Salicylaldehyde is produced by hydrolysis of plant derived salicin to salicyl alcohol, followed by oxidation to salicyladehyde (Hilker and Schulz 1994). Another species, Chrysomela lapponica, shows population variation in their chemistry. Populations associated with salicin-poor willows or birches do not produce salicylaldehyde whereas populations associated with salicin-rich willows do (Gross and Hilker 1994; Geiselhardt et al. 2015). Predators, both carnivorous sawfly larvae (Hymenoptera: Tenthredo; Pasteels and Gregoire 1984) and ants (Hymenoptera: Formica; Zvereva et al. 2016) are initially repelled by the larval secretion but can both overcome the repulsion with experience, indicating that the defence may be most effective when predation levels are relatively low. It is not only the larvae that are chemically defended as some species, for instance $P$. vitellinae sequester salicin in their eggs which is an effective deterrent to ant predation (Pasteels et al. 1986). However, defence is not the only effect of these secretions as they also regulate conspecific and interspecific intergenerational competition by deterring feeding and oviposition by adults of the same species as well as other chrysomelid species (Hilker 1989). This anti-competitive effect may be as important as the defence role, if not more important.

Fourthly, the willow-feeding chrysomelids form host races with distinctive host specificity. The example was given above of substantial differences in biochemistry between populations of $C$. lapponica (particularly the ability to use salicin as a substrate). This is not the only example of recent evolution in the group. Particular interest attaches to Lochmaea capreae (Linnaeus, 1758), which like C. lapponica has willow and birch associated populations, but in this case they are sympatric (Soudi et al. 2015). The host-specific populations of $L$. capreae have been shown to have a genetic basis and to be true host 
races (Soudi et al. 2016). An intriguing example of active evolution is provided by Plagiodera versicolora (Laicharting, 1781), in which populations are under selection either for feeding exclusively on new leaves (gourmet populations) or on all leaves (no preference populations) (Utsumi et al. 2012, Utsumi et al. 2009). In this instance the feeding preference feeds back via plant response to the herbivory to have a profound effect on the willow-associated arthropod community composition and dynamics. For instance gourmet feeding by chrysomelids resulted in more aphids (Utsumi 2015).

Fifthly, the willow-feeding chrysomelids are prone to outbreaks and thus have an interesting and dynamic population biology. For instance a study of $P$. vulgatissima on Salix viminalis in Ireland (Kelly and Curry 1991a) showed a variation in successive years from maximal mean densities of 308 adults per tree to 72 adults per tree the following year. A study of the same species found that beetle density was lower in mixed species willow stands than in monocultures (Peacock and Herrick 2000). Chrysomelid populations are regulated by predators including heteropteran bugs such as Anthocoris and parasitoids. Herbivory by $P$. vulgatissima has been shown to attract Anthocoris (Lehrman et al. 2013). The parasitoid wasp, Perilitus brevicollis Haliday 1835, also attacks Phratora vulgatissima. However, somewhat paradoxically, control is limited at high beetle densities, as at high densities beetles become smaller, which causes parasitoid survival to decrease (Stenberg 2015).

Sixthly, it should be noted that many willow-feeding chrysomelids have highly temperature dependent development and thus should be highly responsive to interannual climatic variation and, ultimately, to climate change. Perhaps related to this, chrysomelids are known to have distinctive distribution patterns within Europe (Schmitt and Rönn 2011). Kutcherov (2015) has shown that Chrysomela vigintipunctata requires 275.5 degree days (DD) above a threshold of $9.0^{\circ} \mathrm{C}$ for egg to adult development. In cold weather the adults appear later and are larger (as development has been slower). In warmer weather adults appear sooner and are smaller (having developed fast). Changes in beetle distribution, phenology or size with changing temperature may in turn have knock-on effects on other willow-associated arthropod communities, and perhaps thereby on whole ecosystems.

Most studies involving willow-feeding chrysomelids are specific to a single locality or geographical region. We wished to determine the most abundant species of willowassociated chrysomelids over a wide geographical range and to assess their patterns of occurrence and co-occurrence, and their population variability as part of a broader study on willow communities across Europe. Therefore chrysomelid beetles were collected by one of us (ER) from 41 willow stands over a north-south megatransect from Greece to Arctic Norway. This megatransect has been described previously (Cronk et al. 2015). 


\section{Materials and Methods}

\section{Site selection and details}

Full details of the sites and their selection have been given previously (Cronk et al. 2015). Briefly the route from Greece to Arctic Norway was driven in 2015, stopping approximately every $100 \mathrm{~km}$ to locate and sample a stand of willows (Salix spp.) (Table 1).

\section{Table 1.}

Site details. Further information can be found in Cronk et al. (2015).

\begin{tabular}{|c|c|c|c|c|}
\hline SITE\# & Country & Lat $N$ & Long E & Alt (m) \\
\hline 1 & Greece & 38.80007 & 22.4629 & 37 \\
\hline 2 & Greece & 38.902 & 22.31015 & 33 \\
\hline 3 & Greece & 39.306694 & 22.528323 & 177 \\
\hline 4 & Greece & 40.032685 & 22.175437 & 534 \\
\hline 5 & Greece & 41.113317 & 23.273893 & 31 \\
\hline 6 & Bulgaria & 41.412468 & 23.318609 & 90 \\
\hline 7 & Bulgaria & 42.165622 & 22.998141 & 392 \\
\hline 8 & Bulgaria & 42.923989 & 23.810563 & 339 \\
\hline 9 & Bulgaria & 43.739343 & 23.966755 & 35 \\
\hline 10 & Romania & 44.260343 & 23.786781 & 81 \\
\hline 11 & Romania & 44.961981 & 23.190337 & 172 \\
\hline 12 & Romania & 45.510676 & 22.737225 & 556 \\
\hline 13 & Romania & 46.518504 & 21.512839 & 102 \\
\hline 14 & Hungary & 46.700744 & 21.31268 & 94 \\
\hline 15 & Hungary & 47.665648 & 21.261768 & 91 \\
\hline 16 & Hungary & 48.374291 & 20.725264 & 148 \\
\hline 17 & Poland & 49.463447 & 21.697255 & 385 \\
\hline 18 & Poland & 50.470234 & 22.238372 & 157 \\
\hline 19 & Poland & 50.673994 & 21.823391 & 141 \\
\hline 20 & Poland & 51.775039 & 21.1971 & 101 \\
\hline 21 & Poland & 52.69398 & 21.8529 & 96 \\
\hline 22 & Poland & 53.55483 & 22.30299 & 128 \\
\hline 23 & Poland & 54.06943 & 23.11745 & 137 \\
\hline
\end{tabular}




\begin{tabular}{|c|c|c|c|c|}
\hline 24 & Lithuania & 54.92583 & 23.7742 & 28 \\
\hline 25 & Lithuania & 55.79557 & 24.56678 & 62 \\
\hline 26 & Latvia & 56.71141 & 24.25162 & 23 \\
\hline 27 & Latvia & 57.74963 & 24.4023 & 7 \\
\hline 28 & Estonia & 58.42257 & 24.44063 & 18 \\
\hline 29 & Estonia & 59.40289 & 24.93577 & 48 \\
\hline 30 & Finland & 60.27299 & 24.65843 & 33 \\
\hline 31 & Finland & 61.09965 & 25.6282 & 84 \\
\hline 32 & Finland & 62.04962 & 26.12369 & 174 \\
\hline 33 & Finland & 63.01589 & 25.80457 & 139 \\
\hline 34 & Finland & 64.05074 & 25.52664 & 91 \\
\hline 35 & Finland & 64.61287 & 25.53805 & 58 \\
\hline 36 & Finland & 65.32835 & 25.29175 & 1 \\
\hline 37 & Finland & 66.24947 & 23.8945 & 51 \\
\hline 38 & Finland & 67.21253 & 24.12629 & 160 \\
\hline 39 & Finland & 67.91183 & 23.63411 & 233 \\
\hline 40 & Norway & 68.8138 & 23.26658 & 374 \\
\hline 41 & Norway & 69.72487 & 23.40581 & 289 \\
\hline
\end{tabular}

\section{Collecting methods}

Willow associated beetles were collected at every site. A sweep net was used with an attempt to sample from all the taxa of willows present at a site. Willows commonest at a site were sampled more. Sampling duration was approximately 1 hour per site. An attempt was made to separate collections from each species of willow, but as field identification of willows is often difficult and complicated by hybridization this was not always possible. For the purposes of this paper all samples at a site are pooled. The willows at each site and voucher herbarium specimens are given elsewhere (Cronk et al. 2015). Beetle samples were immediately transferred, in the field, into tubes containing $70 \%$ alcohol. Alcohol preserved material was then kept at ambient temperature and transferred to the $\mathrm{NHM}$ (London) for subsequent sorting. As collecting efficiency may be influenced by environmental conditions the time of day, relative humidity $(\mathrm{rH})$ and temperature $\left(\mathrm{t}^{\circ} \mathrm{C}\right)$ were also recorded for each site (Table 2; Fig. 1). Relative humidity and temperature were recorded using a Hyelec MS6508 thermohygrometer. 
Table 2.

Date, time and environmental conditions at the start of collection.

\begin{tabular}{|c|c|c|c|c|}
\hline SITE & temp C & humidity $\%$ & time & date \\
\hline 1 & 23 & 27 & 13.35 & 21-iv-2015 \\
\hline 2 & 20 & 37 & 16.4 & 21-iv-2015 \\
\hline 3 & 19.5 & 33 & 12.1 & 22-iv-2015 \\
\hline 4 & 21 & 25 & 17.05 & 22-iv-2015 \\
\hline 5 & 17 & 53 & 12.25 & 23-iv-2015 \\
\hline 6 & 21.5 & 40 & 17 & 23-iv-2015 \\
\hline 7 & 15.2 & 56 & 10.3 & 24-iv-2015 \\
\hline 8 & 24.3 & 24 & 16.3 & 24-iv-2015 \\
\hline 9 & 21.5 & 44 & 19.05 & 24-iv-2015 \\
\hline 10 & 24.5 & 36 & 13.05 & 25-iv-2015 \\
\hline 11 & 24 & 30 & 16.3 & 25-iv-2015 \\
\hline 12 & 19.5 & 43 & 10.25 & 26-iv-2015 \\
\hline 13 & 21 & 44 & 18.05 & 26-iv-2015 \\
\hline 14 & 22 & 47 & 10.3 & $27-i v-2015$ \\
\hline 15 & 26 & 40 & 16.3 & 27-iv-2015 \\
\hline 16 & 19.5 & 53 & 11.5 & 28-iv-2015 \\
\hline 17 & 18.9 & 54.5 & 18 & 28 -iv-2015 \\
\hline 18 & 12.5 & 52 & 12 & 29-iv-2015 \\
\hline 19 & 17.5 & 50 & 15 & 29-iv-2015 \\
\hline 20 & 12.7 & 48 & 9 & $30-i v-2015$ \\
\hline 21 & 27.2 & 32 & 12.3 & $12-v i-2015$ \\
\hline 22 & 26.7 & 36 & 17.15 & $12-v i-2015$ \\
\hline 23 & 22.3 & 74 & 10 & 13-vi-2015 \\
\hline 24 & 26.5 & 56 & 14.45 & 13-vi-2015 \\
\hline 25 & 22.9 & 65 & 19.4 & 13-vi-2015 \\
\hline 26 & 24.3 & 60 & 10 & 14-vi-2015 \\
\hline 27 & 20.8 & 77 & 15.45 & $14-v i-2015$ \\
\hline 28 & 16.2 & 77 & 8.3 & $15-v i-2015$ \\
\hline
\end{tabular}




\begin{tabular}{|c|c|c|c|c|}
\hline 29 & 13.8 & 66 & 13.4 & $15-v i-2015$ \\
\hline 30 & 13.3 & 46 & 10.3 & $16-v i-2015$ \\
\hline 31 & 14.5 & 47 & 16 & $16-v i-2015$ \\
\hline 32 & 13.8 & 54 & 10.45 & $17-v i-2015$ \\
\hline 33 & 16.3 & 48 & 15 & $17-v i-2015$ \\
\hline 34 & 13.9 & 53 & 19 & $17-v i-2015$ \\
\hline 35 & 15.3 & 53 & 12 & $18-v i-2015$ \\
\hline 36 & 16.9 & 43 & 16 & $18-v i-2015$ \\
\hline 37 & 16.5 & 49 & 10.15 & $19-v i-2015$ \\
\hline 38 & 16.1 & 44 & 14.3 & $19-v i-2015$ \\
\hline 39 & 13.3 & 40 & 18.15 & $19-v i-2015$ \\
\hline 40 & 15.1 & 38 & 11.3 & $20-v i-2015$ \\
\hline 41 & 14.8 & 49 & 15.45 & $20-v i-2015$ \\
\hline
\end{tabular}

Table 3.

Species recorded, in order of number of sites. The first 8 species are the most widespread and have sufficient representation to be classified into wide, northern and southern occurrence tendencies.

\begin{tabular}{|l|l|l|l|l|}
\hline SPECIES & $\begin{array}{l}\text { Number of } \\
\text { Sites (S) }\end{array}$ & $\begin{array}{l}\text { No. of Individuals } \\
\text { (N) }\end{array}$ & $\begin{array}{l}\text { Abundance index } \\
\text { (NxS) }\end{array}$ & Site Range \\
\hline $\begin{array}{l}\text { Crepidodera aurata Marsham, } \\
1802\end{array}$ & 27 & $>267$ & 7209 & 3 - 39 [Wide] \\
\hline $\begin{array}{l}\text { Phratora vitellinae (Linnaeus, } \\
\text { 1758) }\end{array}$ & 21 & $>215$ & 4515 & 7 - 41 [Wide] \\
\hline $\begin{array}{l}\text { Crepidodera fulvicornis } \\
\text { (Fabricius, 1792) }\end{array}$ & 19 & 191 & 3629 & (11-)23-39 \\
\hline $\begin{array}{l}\text { Galerucella lineola (Fabricius, } \\
\text { 1781) }\end{array}$ & 19 & $>267$ & 5073 & 11 - 39 [Wide] \\
\hline $\begin{array}{l}\text { Plagiodera versicolora } \\
\text { (Laicharting, 1781) }\end{array}$ & 11 & 43 & 473 & 6 [-20(-39) \\
\hline $\begin{array}{l}\text { Chrysomela vigintipunctata } \\
\text { Scopoli, 1763 }\end{array}$ & 9 & 34 & 306 & 4 - 25 [Southern] \\
\hline $\begin{array}{l}\text { Crepidodera plutus (Latreille, } \\
\text { 1804) }\end{array}$ & 9 & $>57$ & 513 & 9 [Southern] \\
\hline
\end{tabular}




\begin{tabular}{|c|c|c|c|c|}
\hline $\begin{array}{l}\text { Gonioctena pallida (Linnaeus, } \\
1758 \text { ) }\end{array}$ & 8 & $>90$ & 720 & 32 - 41 [Northern] \\
\hline Altica sp. & 4 & 7 & 28 & $6,8,22,23$ \\
\hline $\begin{array}{l}\text { Chrysomela populi Linnaeus, } \\
1758\end{array}$ & 3 & 5 & 15 & $12,13,17$ \\
\hline $\begin{array}{l}\text { Crepidodera aurea (Geoffroy, } \\
1785 \text { ) }\end{array}$ & 3 & 8 & 24 & $12,30,32$ \\
\hline Cryptocephalus sp. & 3 & 11 & 33 & $3,6,24$ \\
\hline $\begin{array}{l}\text { Phratora vulgatissima (Linnaeus, } \\
\text { 1758) }\end{array}$ & 3 & 15 & 45 & $15,18,39$ \\
\hline Agelastica alni (Linnaeus, 1758) & 2 & 2 & 4 & 28,30 \\
\hline $\begin{array}{l}\text { Chaetocnema picipes Stephens, } \\
1831\end{array}$ & 2 & 2 & 4 & 11,18 \\
\hline Chaetocnema sp. & 2 & 2 & 4 & 11,23 \\
\hline $\begin{array}{l}\text { Cryptocephalus decemaculatus } \\
\text { (Linnaeus, 1758) }\end{array}$ & 2 & 2 & 4 & 25,28 \\
\hline Dibolia sp. & 2 & 3 & 6 & 6,7 \\
\hline $\begin{array}{l}\text { Gonioctena linnaeana Schrank, } \\
1781\end{array}$ & 2 & 4 & 8 & 38,39 \\
\hline $\begin{array}{l}\text { Gonioctena viminalis (Linnaeus, } \\
1758 \text { ) }\end{array}$ & 2 & 14 & 28 & 33,37 \\
\hline $\begin{array}{l}\text { Lochmaea caprea (Linnaeus, } \\
1758 \text { ) }\end{array}$ & 2 & 9 & 18 & 25,26 \\
\hline Longitarsus sp. & 2 & 2 & 4 & 11,27 \\
\hline $\begin{array}{l}\text { Smaragdina salicina (Scopoli, } \\
1763 \text { ) }\end{array}$ & 2 & 2 & 4 & 12,13 \\
\hline $\begin{array}{l}\text { Bromius obscurus (Linnaeus, } \\
1758 \text { ) }\end{array}$ & 1 & 4 & 4 & 33 \\
\hline $\begin{array}{l}\text { Chrysolina fastuosa Scopoli, } \\
1763\end{array}$ & 1 & 2 & 2 & 1 \\
\hline $\begin{array}{l}\text { Chrysolina graminis Linnaeus, } \\
1758\end{array}$ & 1 & 2 & 2 & 37 \\
\hline $\begin{array}{l}\text { Cryptocephalus sexpunctatus } \\
\text { (Linnaeus, 1758) }\end{array}$ & 1 & 5 & 5 & 11 \\
\hline $\begin{array}{l}\text { Cryptocephalus exiguus } \\
\text { Schneider, } 1792\end{array}$ & 1 & 3 & 3 & 24 \\
\hline
\end{tabular}




\begin{tabular}{|l|l|l|l|l|}
\hline Donacia aquatica Kunze, 1818 & 1 & 1 & 1 & 38 \\
\hline Donacia simplex Fabricius, 1775 & 1 & 1 & 1 & 29 \\
\hline $\begin{array}{l}\text { Gonioctena nivosa (Suffian, } \\
1851)\end{array}$ & 1 & 1 & 1 & 33 \\
\hline $\begin{array}{l}\text { Lytharia salicariae (Paykull, } \\
1800)\end{array}$ & 1 & 2 & 2 & 26 \\
\hline Phratora laticollis Suffian, 1851 & 1 & 18 & 18 & 11 \\
\hline $\begin{array}{l}\text { Smaragdina flavicollis } \\
\text { Charpentier, 1825 }\end{array}$ & 1 & 1 & 1 & 28 \\
\hline
\end{tabular}

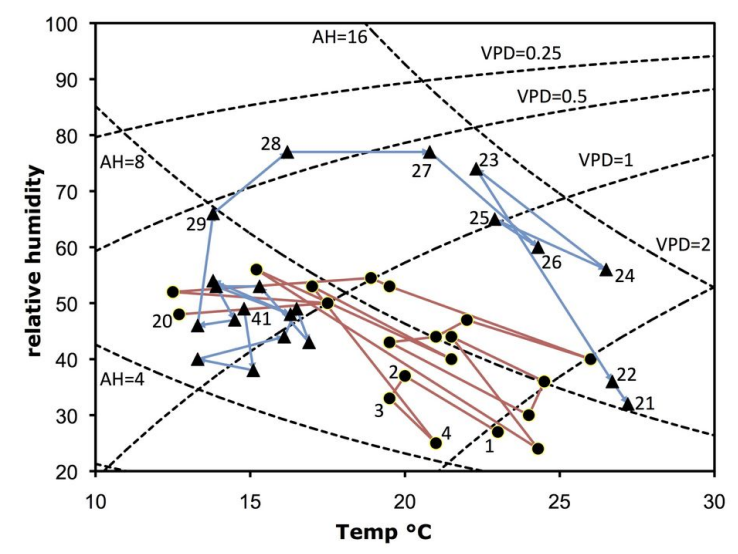

Figure 1.

Collecting conditions (temperature and relative humidity) at the sites (data plotted from Table 2). In this graph lines of constant absolute humidity $\left(\mathrm{AH} ; \mathrm{g} / \mathrm{m}^{3}\right)$ and vapour pressure deficit (VPD; $\mathrm{kPa}$ ) are plotted as dashed lines. VPD is a measure of the drying power of the air. Circles (red line) mark collection localities 1-20 (April 2015) while the triangles (blue line) mark sites 21-41 (June 2015). Note that the environmental conditions during collection are very similar between Central Europe (sites 18-20) in April and Arctic Europe (sites 30-41) in June.

\section{Specimen examination and analysis}

Specimens from each locality were sorted into broad morphospecies, identified and counted. Identifications were made by RC. Most morphospecies likely correspond to biological species. The following works and resources were consulted for the identification of taxa: Hubble (2012); Borowiec (2013); Warchałowski (2003); Warchałowski (2010); Lompe (2002); Watford Coleoptera Group (2016); and the species list of Volf et al. (2015). Some of the most abundant species (Crepidodera aurata Marsham, 1802, C. fulvicornis (Fabricius, 1792), C. plutus (Latreille, 1804), Phratora vitellinae (Linnaeus, 1758), Galerucella lineola (Fabricius, 1781), Plagiodera versicolora (Laicharting, 1781), 
Chrysomela vigintipunctata Scopoli, 1763 and Gonioctena pallida (Linnaeus, 1758)) were subsampled (one to three individuals per sample from 6 samples per species) from selected localities for imaging and measurement. Measurements were performed using a Zeiss Stemi DV4 dissecting scope and a Minitool miniature measuring scale with a $5 \mathrm{~mm}$ range calibrated to $0.1 \mathrm{~mm}$. Colours were determined by matching to the standard RHS colour chart (RHS 2001). Colour codes were translated to colour names using standard practice (UPOV 2013). Photographs were taken using a Canon EOS 700D, viewing through a Leica MZ12.5 stereomicroscope. Photos were taken via a Dell computer using the Canon EOS 700D Utility Remote Live View programme to take several photos of each specimen at different focus distances. These photos were then combined together to form a fully focused image using the focus stacking software Helicon Focus (version 5.3).

\section{Data Analysis}

The inter-site latitudinal variation in occurrence of the eight commonest species (Table 4) was examined using Canonical Principal Components Analysis (Redundancy Analysis), with latitude as the explanatory variable. The beetle matrix of counts of individuals (Table 4) was square root transformed to normalise. The beetle matrix was used as the response matrix. Redundancy analysis was performed using the Java package Ginkgo in the software suite B-VegAna (Font Castell 2006).

Table 4.

Abundance of common species at sites. Counts of individuals are given for all samples. Abbreviations: Ch. vig. $=$ Chrysomela vigintipunctata Scopoli, $1763 ;$ Cr. aura $=$ Crepidodera aurata Marsham, 1802; Cr. fulv. = Crepidodera fulvicornis (Fabricius, 1792); Cr. plutus = Crepidodera plutus (Latreille, 1804); G. lineo. = Galerucella lineola (Fabricius, 1781); Gonio. pal. = Gonioctena pallida (Linnaeus, 1758); Ph. vitel. $=$ Phratora vitellinae $($ Linnaeus, 1758$) ;$ Pl. vers. $=$ Plagiodera versicolora (Laicharting, 1781); Tot $(\mathrm{com})=$ Total individuals at sites (common species); Tot (all) = Total individuals at sites (all species); N. spp. = number of chrysomelid species at sites. Counts marked $>$ indicate that not all individuals were counted.

\begin{tabular}{|c|c|c|c|c|c|c|c|c|c|c|c|}
\hline Site & $\begin{array}{l}\text { Ch. } \\
\text { vig. }\end{array}$ & $\begin{array}{l}\text { Cr. } \\
\text { aura. }\end{array}$ & $\begin{array}{l}\text { Cr. } \\
\text { fulv. }\end{array}$ & $\begin{array}{l}\text { Cr. } \\
\text { plutus }\end{array}$ & $\begin{array}{l}\text { G. } \\
\text { lineo. }\end{array}$ & $\begin{array}{l}\text { Gonio. } \\
\text { pal. }\end{array}$ & $\begin{array}{l}\text { Ph. } \\
\text { vit. }\end{array}$ & PI. vers. & $\begin{array}{l}\text { Tot. } \\
\text { (com) }\end{array}$ & $\begin{array}{l}\text { Tot. } \\
\text { (all) }\end{array}$ & $\begin{array}{l}\text { N. } \\
\text { spp }\end{array}$ \\
\hline 1 & & & & & & & & & 0 & 1 & 1 \\
\hline 2 & & & & & & & & & 0 & 0 & 0 \\
\hline 3 & & 2 & & & & & & & 2 & 3 & 2 \\
\hline 4 & 1 & 6 & & & & & & & 7 & 7 & 2 \\
\hline 5 & & & & & & & & & 0 & 0 & 0 \\
\hline 6 & 1 & 1 & & & & & & 11 & 13 & 18 & 6 \\
\hline 7 & 2 & 4 & & & & & 16 & 5 & 27 & 29 & 5 \\
\hline 8 & & 30 & & & & & & & 30 & 31 & 2 \\
\hline
\end{tabular}




\begin{tabular}{|c|c|c|c|c|c|c|c|c|c|c|c|}
\hline 9 & & & & 9 & & & & 1 & 10 & 10 & 2 \\
\hline 10 & & 2 & & 4 & & & & 3 & 9 & 9 & 3 \\
\hline 11 & 4 & 32 & 1 & 3 & 9 & & 2 & 1 & 52 & 78 & 12 \\
\hline 12 & & 15 & & 3 & & & & 3 & 21 & 26 & 6 \\
\hline 13 & 1 & 6 & & 3 & & & & & 10 & 13 & 5 \\
\hline 14 & & 12 & & 7 & 1 & & & & 20 & 20 & 3 \\
\hline 15 & & 6 & & & & & 2 & & 8 & 10 & 3 \\
\hline 16 & 10 & 22 & 1 & & 1 & & 1 & 6 & 41 & 41 & 6 \\
\hline 17 & 3 & $>40$ & & & & & 20 & & 63 & 64 & 4 \\
\hline 18 & & $>20$ & & & 9 & & & 1 & 30 & 38 & 5 \\
\hline 19 & & 4 & 4 & $>20$ & 5 & & & & 33 & 33 & 4 \\
\hline 20 & & 2 & & & 1 & & 3 & 7 & 13 & 13 & 4 \\
\hline 21 & 1 & & & 7 & & & & & 8 & 8 & 2 \\
\hline 22 & & 4 & & & & & & & 4 & 5 & 2 \\
\hline 23 & & & 26 & 1 & 7 & & & & 34 & 39 & 6 \\
\hline 24 & & & & & & & & & 0 & 10 & 2 \\
\hline 25 & 11 & 15 & 7 & & & & 1 & & 34 & 36 & 6 \\
\hline 26 & & 1 & 11 & & 9 & & $>20$ & & 41 & 51 & 6 \\
\hline 27 & & 3 & 4 & & & & 3 & & 10 & 11 & 4 \\
\hline 28 & & 12 & 19 & & & & 24 & & 55 & 58 & 6 \\
\hline 29 & & 6 & 1 & & 3 & & 1 & & 11 & 13 & 6 \\
\hline 30 & & 2 & 17 & & 8 & & 3 & & 30 & 32 & 6 \\
\hline 31 & & & 12 & & 3 & & 19 & & 34 & 34 & 3 \\
\hline 32 & & & 16 & & 22 & 1 & 26 & & 65 & 70 & 5 \\
\hline 33 & & 1 & 8 & & 8 & & 9 & 3 & 29 & 39 & 8 \\
\hline 34 & & & 11 & & 25 & 1 & 5 & & 42 & 42 & 4 \\
\hline 35 & & & 34 & & $>50$ & 1 & 1 & & 86 & 86 & 4 \\
\hline 36 & & 2 & 10 & & $>40$ & 1 & 6 & & 59 & 59 & 5 \\
\hline 37 & & 2 & 5 & & 5 & 6 & 18 & & 36 & 47 & 7 \\
\hline 38 & & 10 & 1 & & $>50$ & & 27 & & 88 & 90 & 6 \\
\hline 39 & & 5 & 3 & & 11 & 10 & & 2 & 31 & 40 & 7 \\
\hline
\end{tabular}




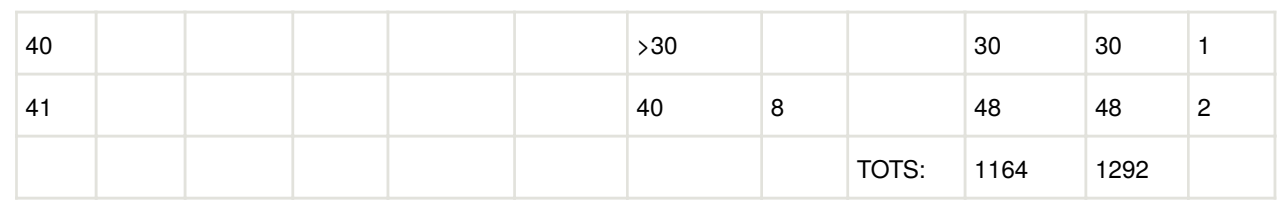

\section{Results}

\section{Species encountered and their relative abundance}

The list of species encountered is given in Table 3. The sites along the transect yielded 34 morphospecies of willow-associated chrysomelid. The most widespread and abundant of these was $C$. aurata, which occured at 27 out of 41 sites and $>267$ individuals were captured in our samples. In all, eight morphospecies were common, occurring at eight or more sites and in considerable abundance (Table 3). The remaining 26 morphospecies were comparatively sparsely distributed with 11 being found at a single site only. The eight common species contributed 1164 counted individuals in our samples. The remaining 26 morphospecies contributed only a further 128 counted individuals (Table 3). Most of the species are known willow-feeders. However, some species taken from willow are commonly recorded as feeding exclusively on other types of plant (Böhme 2001): Donacia aquatica (Carex spp.), D. simplex (Sparganium spp.) and Chrysolina graminis (Asteraceae). Nevertheless they are included here as willow-associated, and examples of beetles that may be taken as by-catch when sampling willows. Site descriptions (Cronk et al. 2015) for sites 29 and 38, where Donacia is present, indicate their suitability (as wetland sites) for these species.

Chrysomelids were rare in Greece and were absent from two Greek sites sampled (2 \& 5). However, they were generally abundant at all other sites (from Bulgaria to Norway) (Table 4). The mean number of captured and counted individuals at Greek sites was 2.2 with a range of 0-7. For the remaining sites the mean was 35.6 (range 5-90) (Table 4).

In terms of number of morphospecies per site, Greek sites had an average of 1 species (range $0-2$ ) and the other sites an average of 4.7 species (range 2-12) (Table 3). All sites together had an average number of species per site of 4.2 .

\section{Geographical Patterns in the commonest species}

The commonest species and their site distributions are detailed in Table 4. The species showed clear evidence of geographical patterning (Table 4). Of the eight abundant species, three were very widespread along the transect (C. aurata, $P$. vitellinae and $G$. lineola). Two had northern-biased distributions ( $C$. fulvicornis and Gonioctena pallida) and three southern-biased distributions (P. versicolora, $C$. vigintipunctata and $C$. plutus). 


\section{Correlations with latitude}

Redundancy analysis showed that variation in occurrence of chrysomelids (common species) was, as expected, highly correlated with latitude. Latitude was able to explain $23.2 \%$ of the total variance in the beetle matrix. When the latitude input order was randomized multiple times, latitude was only able to explain around $2 \%$ of the variance by chance alone $($ mean $=2.26 \%$, standard deviation $=0.71)$. However this correlation with latitude is mainly due to (1) the paucity chrysomelids at the southernmost sites (Greece) and (2) the difference between a distinctly boreal chrysomelid fauna north of Poland contrasting with a rather homogeneous central European fauna from Bulgaria to Poland (sites 6 to 23). When sites 6 to 23 are analyzed separately there is little association with latitude (6.8\%) and this is not much better than random (random: $3.66 \%$, SD 1.36).

\section{Morphological Variation}

We noted considerable variation in colour and size of the common beetles from population to population but within populations they tended to be fairly homogeneous. All the common species displayed great chromatic variation (Table 5; Fig. 2; Fig. 3) with the exception of G. lineola. In this species no variation in colour was detectable by the human eye. Species also differed in their size variation: most were quite variable between populations but the three species of Crepidodera were comparatively invariant in size (Table 5).

\section{Table 5.}

Measurements of six to eight representative individuals of the common Chrysomelids (one to three per site) chosen to show variation.

\begin{tabular}{|c|c|c|c|c|c|c|c|}
\hline Species & Sites & $\begin{array}{l}\text { Elytral Colour } \\
\text { on scored } \\
\text { individuals }\end{array}$ & $\begin{array}{l}\text { Main elytral } \\
\text { colours (sites) }\end{array}$ & $\begin{array}{l}\text { Elytral } \\
\text { length } \\
(\mathrm{mm})\end{array}$ & $\begin{array}{l}\text { Elytral } \\
\text { width at } \\
\text { shoulder } \\
(\mathrm{mm})\end{array}$ & $\begin{array}{l}\text { Pronotal } \\
\text { length } \\
(\mathrm{mm})\end{array}$ & $\begin{array}{l}\text { Pronotal } \\
\text { width at } \\
\text { base }(\mathrm{mm})\end{array}$ \\
\hline $\begin{array}{l}\text { Chrysomela } \\
\text { vigintipunctata }\end{array}$ & $\begin{array}{l}4,7 \\
11,16 \\
21,25\end{array}$ & $\begin{array}{l}\text { 161B, 162B, } \\
162 \mathrm{C}, 161 \mathrm{C} \\
155 \mathrm{C}, 155 \mathrm{C}\end{array}$ & $\begin{array}{l}\text { Light yellow } \\
\text { brown }(4,7,11 \text {, } \\
\text { 16); white (21, } \\
25)\end{array}$ & 5.3-6.8 & 3.0-3.7 & 1.4-1.5 & $2.7-3.2$ \\
\hline $\begin{array}{l}\text { Crepidodera } \\
\text { aurata }\end{array}$ & $\begin{array}{l}4,11, \\
18,25, \\
33,39\end{array}$ & $\begin{array}{l}\text { 135B, 118C, } \\
\text { 119B, 118C, } \\
118 \mathrm{~B}, 111 \mathrm{~B}\end{array}$ & $\begin{array}{l}\text { dark green }(4) ; \\
\text { light green blue } \\
(11,25) ; \text { grey } \\
\text { blue }(18) ; \text { green } \\
\text { blue }(33,39)\end{array}$ & 2.0-2.2 & $1.2-1.2$ & $0.5-0.6$ & $1.0-1.1$ \\
\hline $\begin{array}{l}\text { Phratora } \\
\text { vitellinae }\end{array}$ & $\begin{array}{l}7,15, \\
20,26, \\
32,41\end{array}$ & $\begin{array}{l}\text { 111A, 111A, } \\
\text { 111B, 137B, } \\
\text { N144A, 146D }\end{array}$ & $\begin{array}{l}\text { green blue }(7, \\
15,20) ; \text { brown } \\
\text { green }(26,41) ; \\
\text { dark green (32) }\end{array}$ & 3.5-3.9 & $1.9-2.4$ & $1-1.1$ & $1.5-2.0$ \\
\hline
\end{tabular}




\begin{tabular}{|c|c|c|c|c|c|c|c|}
\hline $\begin{array}{l}\text { Plagiodera } \\
\text { versicolora }\end{array}$ & $\begin{array}{l}6,12, \\
16,20, \\
20,20, \\
33,39\end{array}$ & $\begin{array}{l}\text { 111B, 118B, } \\
\text { 113B, 113B, } \\
\text { N80B, N87B, } \\
\text { 120B, 113B }\end{array}$ & $\begin{array}{l}\text { green blue (6, } \\
12,16,20,39) ; \\
\text { violet }(20) \text {; light } \\
\text { blue green (33) }\end{array}$ & 2.9-3.9 & 2.3-2.5 & $0.9-0.9$ & $1.9-2.2$ \\
\hline $\begin{array}{l}\text { Crepidodera } \\
\text { fulvicornis }\end{array}$ & $\begin{array}{l}16,23, \\
23,23, \\
27,31, \\
35,39\end{array}$ & $\begin{array}{l}\text { 137B, 104B, } \\
\text { 175D, N144B, } \\
\text { 144B, 143B, } \\
\text { N144B, 143C }\end{array}$ & $\begin{array}{l}\text { brown green } \\
(16) ; \text { medium } \\
\text { blue (23); } \\
\text { medium brown } \\
(23) ; \text { light green } \\
(23,27,35) \text {; dark } \\
\text { green }(31,39)\end{array}$ & $1.7-2.2$ & $1-1.3$ & $0.5-0.6$ & $0.8-1.1$ \\
\hline $\begin{array}{l}\text { Galerucella } \\
\text { lineola }\end{array}$ & $\begin{array}{l}7,11 \\
19,26 \\
34,39\end{array}$ & $\begin{array}{l}165 \mathrm{~A}, 165 \mathrm{~A}, \\
165 \mathrm{~A}, 165 \mathrm{~A}, \\
165 \mathrm{~A}, 165 \mathrm{~A}\end{array}$ & $\begin{array}{l}\text { medium brown } \\
\text { (all) }\end{array}$ & $3.5-4.4$ & 1.9-2.2 & $0.8-0.9$ & $1.4-1.5$ \\
\hline $\begin{array}{l}\text { Crepidodera } \\
\text { plutus }\end{array}$ & $\begin{array}{l}9,11 \\
13,14 \\
19,21\end{array}$ & $\begin{array}{l}\text { N144A, N144B, } \\
\text { N144B, 141B, } \\
\text { N144B, 141A }\end{array}$ & $\begin{array}{l}\text { light green }(9,11 \text {, } \\
13,19) ; \text { dark } \\
\text { green }(14,21)\end{array}$ & 2.1-2.4 & 1.2-1.3 & $0.5-0.6$ & $1.0-1.0$ \\
\hline $\begin{array}{l}\text { Gonioctena } \\
\text { pallida }\end{array}$ & $\begin{array}{l}32,34 \\
35,37 \\
39,41\end{array}$ & $\begin{array}{l}\text { 165B, 165B, } \\
\text { 165B, N167A, } \\
\text { N167B, 165B }\end{array}$ & $\begin{array}{l}\text { yellow brown } \\
\text { (all) }\end{array}$ & $3.6-4.8$ & 2.8-3.0 & 1.3-1.3 & $2.6-2.8$ \\
\hline
\end{tabular}

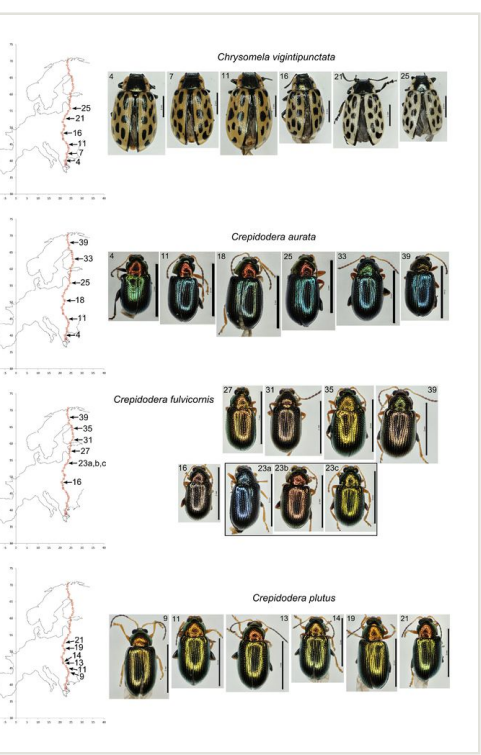

Figure 2 .

Images of representative examples of common species from different populations. Chrysomela vigintipunctata, Crepidodera aurata, C. fulvicornis, C. plutus. Populations are referred to a map (left). Scale bars $=1 \mathrm{~mm}$. 


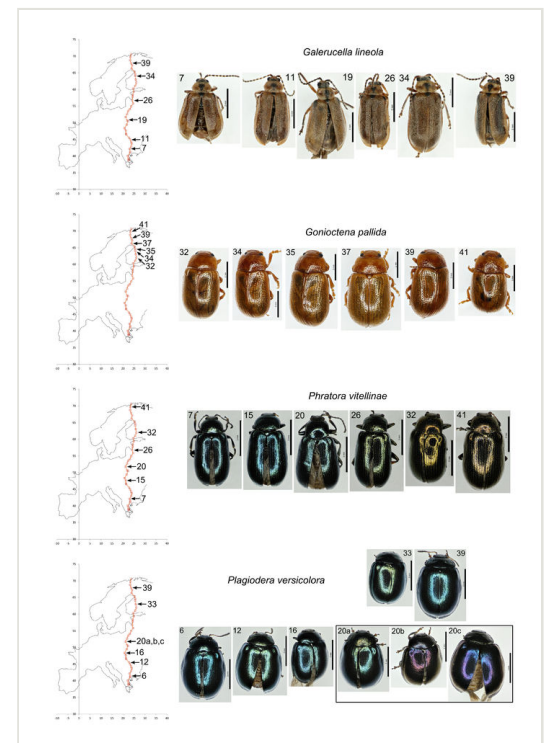

Figure 3.

Images of representative examples of common species from different populations. Galerucella lineola, Gonioctena pallida, Phratora vitellinae, Plagiodera versicolora. Populations are referred to a map (left). Scale bars $=1 \mathrm{~mm}$.

\section{Discussion}

\section{A single-year, time-limited snapshot}

The distribution and abundance of chrysomelids does not just vary geographically. These beetles are well known for temporal variation, both phenological (timing of appearance), population build-up during a year and interannual (year to year) variation driven by episodic outbreaks and population control by parasites and predators. The variation between willow stands, and across Europe will reflect both spatial and temporal patterns. Nevertheless, our "snapshot" of variation gives a clear idea of the variation across Europe to be encountered in a particular year. It also provides the possibility for follow-up specifically to quantify temporal variation. Another advantage of collecting along a geographically wide megatransect is that a full picture of morphological variation within a species is gained (as summarized in Table 5). Biogeographical work in central Europe (Schmitt and Rönn 2011) characterized Crepidodera fulvicornis as "widely distributed", while Gonioctena pallida and Phratora vulgatissima were characterized as "southern", on the basis of 63,000 records. The differences in biogeographical pattern reported here could be due to the "snapshot effect" or simply to the different (more easterly) region being examined. Further work will be needed to distinguish these two hypotheses. 


\section{Potential distributional breakpoints}

It is clear that our sampling reveals a considerable difference between Greece and Bulgaria. This may reflect the comparative rarity of willows in the strongly anthropogenically disturbed and dry Mediterranean climate of Greece, which would deny willow-associated beetles the ready access to this food-plant resource that they have over the rest of Europe. Another possible explanation is that the paucity of Salix-associated chrysomelids in Greece in 2015 is the consequence of phenology or interannual variation (the spring was noted to have been exceptionally warm in Greece in 2015).

Another potential distributional breakpoint we note is around site 23 (northern Poland) which appears to mark a division between the southern-biased common species which end around here (at sites 20-25) and the northern-biased species $C$. fulvicornis which comes in strongly at site 25 (admittedly with southern outliers to site 11). The other northern-biased species, Gonioctena pallida, does not fit the pattern so well, coming in at site 32 (Finland). However this may be due to our late timing of collection with respect to what is clearly a more cryophilous beetle. Generally, the apparent transition point in northern Poland may reflect a genuine biogeographical shift or may simply reflect the particular circumstances of phenology and collection time.

Although this transect was north-south in orientation, the effect of east-west biogeographical boundaries can be seen in the comparative rarity of $P$. vulgatissima ( 3 sites only). This beetle is sometimes stated to be the commonest willow-associated chrysomelid in Europe (as also implied by its Linnaean epithet) so it might appear odd that it was not more abundant in our samples. However it is a species primarily of NW Europe, being particularly abundant in Sweden and Germany westwards to the UK and Norway. Our transect goes through the eastern edge of its range so the comparative rarity in our samples in not surprising.

\section{Acknowledgements}

QCC acknowledges appointments by RBG Kew (as Honorary Research Associate) and by Queen Mary University of London (as Visiting Professor), which greatly facilitated the conduct of this study. We thank Gavin Broad (NHM) for assistance in the field and Rachel Julie-Clark (NHM) for assistance with colour scoring. We are grateful to Michael Schmitt for his helpful comments.

\section{Author contributions}

RC identified and analyzed the beetles and contributed to the writing of the paper; ER collected the beetles and contributed to the analysis and planning of the work and contributed to the writing of the paper; QC wrote the paper and contributed to the analysis 
and planning of the work; DP co-wrote the paper, assisted the analysis, planned and directed the work and obtained funding for the study.

\section{Conflicts of interest}

None

\section{References}

- Böhme J (2001) Phytophage Käfer und ihre Wirtspflanzen in Mitteleuropa. Ein Kompendium. Bioform, Heroldsberg, $132 \mathrm{pp}$.

- Boland W (2015) Sequestration of plant-derived glycosides by leaf beetles: A model system for evolution and adaptation. Perspectives in Science 6: 38-48. DOI: 10.1016/ j.pisc.2015.06.001

- Borowiec L (2013) Website resource of the Department of Biodiversity and Evolutionary Taxonomy, University of Wroclaw. http://culex.biol.uni.wroc.pl/cassidae/European\% 20Chrysomelidae/index.htm. Accession date: 2016512.

- $\quad$ Cronk Q, Ruzzier E, Belyaeva I, Percy D (2015) Salix transect of Europe: latitudinal patterns in willow diversity from Greece to arctic Norway. Biodiversity data journal 3: e6258. DOI: $10.3897 / B D J .3 . e 6258$

- $\quad$ Font Castell X (2006) Ginkgo (B-VegAna). Department of Plant Biology. University of Barcelona. http://biodiver.bio.ub.es/ginkgo/Ginkgo.htm. Accession date: 2016512.

- $\quad$ Geiselhardt S, Hilker M, Müller F, Kozlov MV, Zvereva EL (2015) Inter- and Intrapopulation Variability in the Composition of Larval Defensive Secretions of WillowFeeding Populations of the Leaf Beetle Chrysomela lapponica. Journal of chemical ecology 41: 276-86. DOI: 10.1007/s10886-015-0558-X

- Gross J, Hilker M (1994) Chemoecological studies of the exocrine glandular larval secretions of two chrysomelid species (Coleoptera): Phaedon cochleariae and Chrysomela lapponica . Chemoecology 5: 185-189. DOI: 10.1007/bf01240603

- Haddad S, McKenna D (2016) Phylogeny and evolution of the superfamily Chrysomeloidea (Coleoptera: Cuculiformia). Systematic Entomology 41 (4): 697-716. DOI: $10.1111 /$ syen.12179

- Hilker M (1989) Intra- and interspecific effects of larval secretions in some chrysomelids (Coleoptera). Entomologia Experimentalis et Applicata 53: 237-245. DOI: 10.1111/ j.1570-7458.1989.tb03571.x

- Hilker M, Schulz S (1994) Composition of larval secretion of Chrysomela lapponica (Coleoptera, Chrysomelidae) and its dependence on host plant. Journal of Chemical Ecology 20: 1075-1093. DOI: 10.1007/bf02059744

- Hodkinson ID, Flynn DH, Shackel SC (1998) Relative susceptibility of Salix clones to chrysomelid beetles: evidence from the Stott willow collection at Ness. Forest Pathology 28: 271-279. DOI: 10.1111/j.1439-0329.1998.tb01182.x

- Hubble D (2012) Keys to the adults of seed and leaf beetles of Britain and Ireland. Field Studies Council, Shrewsbury, UK, 136 pp. 
- Jolivet P., Verma K (2002) Biology of Leaf Beetles. Intercept, Andover, 332 pp. [ISBN 1-898298-86-6]

- Jurado-Rivera JA, Vogler AP, Reid CAM, Petitpierre E, Gómez-Zurita J (2009) DNA barcoding insect-host plant associations. Proceedings. Biological sciences / The Royal Society 276: 639-48. DOI: 10.1098/rspb.2008.1264

- Kelly MT, Curry JP (1991a) The influence of phenolic compounds on the suitability of three Salix species as hosts for the willow beetle Phratora vulgatissima . Entomologia Experimentalis et Applicata 61: 25-32. DOI: 10.1111/j.1570-7458.1991.tb02392.x

- $\quad$ Kelly MT, Curry JP (1991b) The biology and population density of the willow beetle ( Phratora vulgatissima [L.]) on Salix viminalis in reclaimed cutaway peat. Journal of Applied Entomology 111: 44-56. DOI: 10.1111/j.1439-0418.1991.tb00293.x

- Kendall DA, Hunter T, Arnold GM, Liggitt J, Morris T, Wiltshire CW (1996) Susceptibility of willow clones (Salix spp.) to herbivory by Phyllodecta vulgatissima (L.) and Galerucella lineola (Fab.) (Coleoptera, Chrysomelidae). Annals of Applied Biology 129: 379-390. DOI: 10.1111/j.1744-7348.1996.tb05762.x

- Kutcherov D (2015) Temperature-dependent development in Chrysomela vigintipunctata (Coleoptera: Chrysomelidae), a stenothermal early-season breeder. Journal of thermal biology 53: 9-14. DOI: 10.1016/j.jtherbio.2015.08.001

- Larsson S, Wirén A (1982) Leaf-eating insects in an energy forest stand of Salix viminalis L. in central Sweden. Annales Entomologici Fennici 48: 119-125.

- Lehrman A, Boddum T, Stenberg JA, Orians CM, Björkman C (2013) Constitutive and herbivore-induced systemic volatiles differentially attract an omnivorous biocontrol agent to contrasting Salix clones. AoB PLANTS 5: plt005. DOI: 10.1093/aobpla/plt005

- Lompe A (2002) Gonioctena in: Käfer Europas. http://www.coleo-net.de/coleo/texte/ gonioctena.htm. Accession date: 2016512.

- $\quad$ Pasteels JM, Gregoire JC (1984) Selective predation on chemically defended chrysomelid larvae : A Conditioning Process. Journal of chemical ecology 10: 1693-700. DOI: $10.1007 / B F 00987355$

- $\quad$ Pasteels JM, Daloze D, Rowell-Rahier M (1986) Chemical defence in chrysomelid eggs and neonate larvae. Physiological Entomology 11: 29-37. DOI: $\underline{10.1111 /}$ j.1365-3032.1986.tb00388.x

- $\quad$ Pasteels JM, Duffey S, Rowell-Rahier M (1990) Toxins in chrysomelid beetles Possible evolutionary sequence from de novo synthesis to derivation from food-plant chemicals. Journal of chemical ecology 16: 211-22. DOI: 10.1007/BF01021280

- $\quad$ Pasteels JM, Rowell-Rahier M, Raupp MJ (1988) Plant-derived defense in chrysomelid beetles. In: Barbosa P, Letourneau D (Eds) Novel aspects of insect-plant interactions. Wiley, New York.

- $\quad$ Pasteels JM, Braekman JC, Daloze D, Ottinger R (1982) Chemical defence in chrysomelid larvae and adults. Tetrahedron 38: 1891-1897. DOI: $10.1016 / 0040-4020$ (82) 80038-0

- Peacock L, Herrick S (2000) Responses of the willow beetle Phratora vulgatissima to genetically and spatially diverse Salix spp. plantations. Journal of Applied Ecology 37: 821-831. DOI: 10.1046/j.1365-2664.2000.00528.x

- RHS (2001) RHS colour chart. Royal Horticultural Society, London, 4 [fans] pp.

- Rowell-Rahier M (1984) The presence or absence of phenolglycosides in Salix (Salicaceae) leaves and the level of dietary specialisation of some of their herbivorous insects. Oecologia 62: 26-30. DOI: $10.1007 /$ bf00377368 
- $\quad$ Royle D, Ostry M (1995) Disease and pest control in the bioenergy crops poplar and willow. Biomass and Bioenergy 9: 69-79. DOI: 10.1016/0961-9534(95)00080-1

- $\quad$ Sage RB, Tucker K (1998) The distribution of Phratora vulgatissima (Coleoptera: Chrysomelidae) on cultivated willows in Britain and Ireland. Forest Pathology 28: 289-296. DOI: 10.1111/j.1439-0329.1998.tb01184.x

- Schmitt M, Rönn T (2011) Types of geographical distribution of leaf beetles (Chrysomelidae) in Central Europe. ZooKeys 157: 131-58. DOI: $10.3897 /$ zookeys.157.1798

- $\quad$ Soudi S, Reinhold K, Engqvist L (2015) Host-associated divergence in sympatric host races of the leaf beetle Lochmaea capreae: implications for local adaptation and reproductive isolation. Biological Journal of the Linnean Society 116: 169-182. DOI: 10. $1111 /$ bij. 12547

- $\quad$ Soudi S, Reinhold K, Engqvist L (2016) Genetic architecture underlying host choice differentiation in the sympatric host races of Lochmaea capreae leaf beetles. Genetica 144: 147-56. DOI: $10.1007 /$ s10709-016-9885-2

- $\quad$ Stenberg J (2015) Outbreaking herbivore escapes parasitoid by attaining only a small body size. Ecosphere 6: art21. DOI: 10.1890/es14-00378.1

- Torp M, Lehrman A, Stenberg JA, Julkunen-Tiitto R, Björkman C (2013) Performance of an herbivorous leaf beetle (Phratora vulgatissima) on Salix F2 hybrids: the importance of phenolics. Journal of chemical ecology 39: 516-24. DOI: 10.1007/s10886-013-0266-3

- $\quad$ UPOV (2013) Glossary of Terms Used in UPOV Documents (Document TGP/14; Associated document to TG/1/3). International Union for the Protection of New Varieties of Plants (UPOV), Geneva, $50 \mathrm{pp}$.

- Utsumi S (2015) Feeding evolution of a herbivore influences an arthropod community through plants: implications for plant-mediated eco-evolutionary feedback loop. Journal of Ecology 103: 829-839. DOI: 10.1111/1365-2745.12419

- Utsumi S, Ando Y, Ohgushi T (2009) Evolution of feeding preference in a leaf beetle: the importance of phenotypic plasticity of a host plant. Ecology Letters 12: 920-929. DOI: 1 0.1111/j.1461-0248.2009.01349.x

- $\quad$ Utsumi S, Ando Y, Roininen H, Takahashi J, Ohgushi T (2012) Herbivore community promotes trait evolution in a leaf beetle via induced plant response. Ecology Letters 16: 362-370. DOI: $10.1111 /$ ele.12051

- Volf M, Hrcek J, Julkunen-Tiitto R, Novotny V (2015) To each its own: differential response of specialist and generalist herbivores to plant defence in willows. The Journal of animal ecology 84: 1123-32. DOI: $10.1111 / 1365-2656.12349$

- Warchałowski A (2003) Chrysomelidae - The leaf-beetles of Europe and the Mediterranean area. Natura Optima Dux Foundation, Warszawa, 599 pp.

- Warchałowski A (2010) The Palaearctic Chrysomelidae. Identification keys. 1 \& 2 . Natura Optima Dux Foundation, Warszawa, 1212 pp.

- Watford Coleoptera Group (2016) Chrysomelid gallery. http://www.thewcg.org.uk/. Accession date: 2016512.

- Z Zvereva E, Kozlov M, Rank N (2016) Does ant predation favour leaf beetle specialization on toxic host plants? Biological Journal of the Linnean Society 119: 201-212. DOI: $\underline{10.1111 / \mathrm{bij} .12801}$ 